\title{
Assessing YouTube as an Educational Tool for Shingles: Cross-Sectional Study
}

Teevit Dunnsiri ${ }^{1}$, BS; Takumi Kawashita ${ }^{1}$, BS; Sharon C Lee ${ }^{1}$, BS; Aaron Kumar Monga ${ }^{1}$, BS; Benjamin K P Woo ${ }^{2}$, MD

${ }^{1}$ Western University of Health Sciences, Pomona, CA, United States

${ }^{2}$ Olive View-UCLA Medical Center, Sylmar, CA, United States

Corresponding Author:

Teevit Dunnsiri, BS

Western University of Health Sciences

309 E 2nd St

Pomona, CA

United States

Phone: 19096236116

Email: teevit@g.ucla.edu

\section{Abstract}

Background: YouTube is a popular platform with many videos, which have potential educational value for medical students. Due to the lack of peer review, other surrogates are necessary to determine the content quality of such educational videos. Few studies have analyzed the research background or academic affiliation of the physicians associated with the production of YouTube videos for medical education. The research background or academic affiliations of those physicians may be a reflection of the content quality of these educational videos.

Objective: This study identifies physicians associated with the production of educational YouTube videos about shingles and analyzes those physicians based on their research background or academic affiliation, which may be good surrogates for video content quality.

Methods: Using the YouTube search engine with default settings, the term "shingles" was searched on May 8, 2020. A cross-sectional study was performed using the first 50 search results. A search on Scopus for each identified physician was performed, and data regarding their research background and academic affiliation were recorded.

Results: Of the 50 YouTube videos, 35 (70\%) were categorized as academic. Of the 35 academic videos, 24 (71\%) videos featured physicians, totaling 25 physicians overall. Out of these 25 physicians, $5(20 \%)$ had at least 1 shingles-related publication and $8(32 \%)$ had an h-index $>10$. A total of $21(84 \%)$ physicians held an academic affiliation.

Conclusions: These results ensure to a certain degree the quality of the content in academic videos on YouTube for medical education. However, further evaluation is needed for this growing platform.

(JMIR Dermatol 2020;3(1):e20338) doi: $\underline{10.2196 / 20338}$

\section{KEYWORDS}

YouTube; medical education; online; shingles; herpes zoster; peer review; dermatology; research background; content quality; views

\section{Introduction}

Shingles, also referred to as herpes zoster, classically presents as a painful vesicular rash distributed along a dermatomal pattern. Symptoms typically last for 2-4 weeks. However, pain can persist even after the rash resolves in a complication known as postherpetic neuralgia. Shingles is caused by reactivation of the varicella-zoster virus, which lies dormant in nerve tissue after resolution of the initial infection known as chickenpox.
Reactivation is typically due to stress or a weakened immunity. Approximately 1 million cases of shingles occur each year in the United States [1]. Despite the introduction of a shingles vaccine in 2006, recent studies have found no significant decline in the incidence of cases [2].

As online media platforms become more pervasive in our society, medical students are finding different methods to incorporate these resources into their education [3]. YouTube is a popular platform with many videos, which have potential 
educational value [4-6]. However, a study on YouTube videos as an educational resource on psoriasis found that misleading videos had a similar number of views to high-quality videos [7]. Although other studies have supported the educational value of YouTube videos, they have also acknowledged the need for more evaluation, particularly via peer-review [8,9].

A different study on psoriasis content on YouTube found that only $7.1 \%$ of the reviewed videos were associated with a medical institution or physician. The study concluded that this emphasized the need for more medical professionals to participate on YouTube in order to raise the quality of information [10]. However, few studies have thoroughly analyzed the physicians associated with the production of YouTube videos for dermatological medical education. Due to the lack of peer-review for YouTube videos, some studies have postulated that the research background of physicians featured in these videos for medical education could be a surrogate for content quality [9]. This study identifies physicians associated with the production of academic YouTube videos about shingles and analyzes their research background and academic affiliation, which may be good surrogates for video content quality.

\section{Methods}

Using the YouTube search engine with default settings, the term "shingles" was searched on May 8, 2020. A cross-sectional study was conducted using the first 50 search results, which were sorted by relevance. Recorded data on the YouTube videos included the category (academic, video blog or "vlog", patient interview, or miscellaneous), number of views, type of health professional associated with the video, and the name of the physician if available. Videos categorized as miscellaneous included commercials and local news reports. An academic video was defined as a video having scientifically correct information about the causes, symptoms, treatment, or prevention of the disease. The accuracy of information from a video was verified by medical students using UpToDate, an evidence-based medical resource. If the presence of a health professional was not clearly discernible in the video, the YouTube channel descriptions of the video producers were investigated.
Scopus is a large abstract and citation database for research. A search on each identified physician was performed on Scopus. Data was recorded from Scopus regarding the searched physicians' area of specialty, h-index, number of publications, academic affiliations, and number of shingles-related publications. The h-index is a measure of the research output and citation impact of an author [11]. The h-index was categorized as either $0-10$ or $>10$.

Physician variables such as having a shingles-related publication, h-index $>10$, or academic affiliation were recorded. Difference in the average number of views between videos associated with a physician who had a shingles-related publication, an h-index $>10$, or an academic affiliation was analyzed. Welch's unpaired $t$ test was utilized due to unequal sample sizes between different groups.

\section{Results}

From the first 50 "shingles" search results on YouTube, there were 35 academic videos, 6 patient interviews, 3 vlogs, and 6 miscellaneous videos. From the 35 academic videos, there were 25 physicians, 1 nurse practitioner, 1 pharmacist, 1 dentist, and 1 medical student identified. A total of 7 academic videos were produced by educational media outlets without an identified health professional. A physician was featured in 2 different academic videos, and 2 academic videos had more than one physician featured. From the 25 physicians identified from academic videos, there were 4 internists, 4 ophthalmologists, 4 family physicians, 3 infectious disease physicians, 3 anesthesiologists, 2 dermatologists, 2 obstetrician-gynecologists, 1 plastic surgeon, 1 urologist, and 1 rheumatologist.

The average number of publications for these 25 physicians was 62 (SD 130). The number of publications ranged from 0 to 529. Among these 25 physicians, 5(20\%) had at least 1 shingles-related publication and $8(32 \%)$ had an h-index $>10$. The h-index had an average of 13 (SD 22) and a range of 0 to 86. A total of $84 \%(21 / 25)$ of the physicians had an academic affiliation (Table 1).

Table 1. Physicians sorted by qualities.

\begin{tabular}{ll}
\hline Physician quality & $\mathrm{n}(\%), \mathrm{N}=25$ \\
\hline Shingles-related publication & $5(20)$ \\
H-index $>10$ & $8(32)$ \\
Academic affiliation & $21(84)$ \\
\hline
\end{tabular}

For academic YouTube videos associated with a verified physician, the average number of views was 9676 (SD 16,888) if there was a shingles-related publication, 59,439 (SD 118,706) if there was an academic affiliation, and 42,191 (SD 184,823) if there was an h-index $>10$. The difference in the average number of views compared to those of videos without these characteristics was not significant (Table 2). Only 4 videos featured physicians who authored a shingles-related publication and only 3 videos featured physicians without an academic affiliation. The total number of views for the 24 academic videos with a verified physician was $1,325,693$. The number of views had a median of 20,554 and an average of 55,237 (SD 111,890). The numbers of views ranged from 231 to 537,390. The video with the most views was associated with an MD-PhD dermatologist who held an academic affiliation. This video alone accounted for $41 \%$ of the total number of views for academic videos with a verified physician. 
Table 2. Comparison of the average number of views by physician characteristics.

\begin{tabular}{llll}
\hline Physician quality & No. of videos & Average no. of views, Mean (SD) & $P$ value \\
\hline $\begin{array}{l}\text { Shingles-related publication } \\
\quad \text { Yes }\end{array}$ & 4 & $9676(16,888)$ & .07 \\
$\quad$ No & 20 & $64,349(120,771)$ & .36 \\
Academic affiliation & & & .57 \\
$\quad$ Yes & 21 & $59,439(118,706)$ & $25,826(39,739)$ \\
No & 3 & & \\
h-index & 16 & $42,191(52,065)$ & \\
$0-10$ & 8 & $81,329(184,823)$ & \\
\hline 10 & &
\end{tabular}

\section{Discussion}

YouTube is one of the world's most popular online media platforms, which many medical students utilize as a resource to supplement their education. Prior studies have suggested the potential benefit that YouTube has for improving medical education. However, those studies also mentioned the need for more evaluations in order to ensure the quality of YouTube videos $[8,9]$. The aim of this study was to analyze the physicians associated with the production of YouTube videos that could be incorporated into medical education. This study was performed around shingles because the unique presentation of this infection makes it well-suited to be studied on online media platforms.

A significant majority of identified physicians had an affiliation with a renowned academic institution with many having produced publications. The substantial research background and academic affiliations of these physicians ensure to a certain degree the accuracy of information in educational YouTube videos about shingles. However, these surrogates for content quality cannot replace the critical analysis that peer-review provides. Preferably, an external rating system should be developed to enable critical analysis by physicians and avoid the limitations that traditional forms of peer-review would impose.

The quality of academic content in a YouTube video may contribute to its popularity. In fact, the video with the most views $(537,390)$ was associated with an MD-PhD dermatologist who has an academic affiliation. This may provide reassurance to viewers given that the associated physician is trained in a specialty that manages patients with shingles. Although videos with physicians who held an academic affiliation or an h-index $>10$ did trend towards more views, this study did not find a significant difference between the number of views and research background of the physicians associated with a video. This suggests that there may be other more significant factors that contribute to the popularity of a video such as the length of time since the video was posted and the number of subscribers of the YouTube channel.

This study has a few limitations that should be considered. The most significant limitation is that the study was unable to characterize the audience of these YouTube videos. Therefore, the proportion of views that could be attributed to medical students could not be gauged. Future studies should utilize YouTube Analytics to survey the audience [12]. Other limitations which also prevented characterization of the audience include YouTube channels turning off the feedback and commenting feature. Moreover, the small sample size in certain categories was a limitation in this study. This limitation was weighed against the decreased relevance of videos as the overall sample size of the study increased. Outside of the first 50 search results were less relevant videos about other topics such as rooftop shingles.

Although this study to a certain degree supports the quality of YouTube videos on shingles for medical education, more evaluation is needed for this growing platform. Future studies should examine how medical students may utilize social media platforms to share such videos. Some studies have suggested that platforms such as WhatsApp and Instagram were highly efficacious in the dissemination of educational information linked to YouTube videos $[13,14]$.

YouTube is an efficient and accessible resource for medical students to supplement their learning. Half of the videos reviewed were associated with verified physicians most of which had an academic affiliation. This study supports to a certain degree the academic quality of YouTube videos about shingles produced for medical education. This provides reassurance to viewers that the information they are receiving is accurate. However, more studies in the future will be needed to assess the utility of YouTube as a tool for medical education.

\section{Conflicts of Interest}

None declared.

\section{References}


1. Insinga RP, Itzler RF, Pellissier JM, Saddier P, Nikas AA. The incidence of herpes zoster in a United States administrative database. J Gen Intern Med 2005 Aug;20(8):748-753 [FREE Full text] [doi: 10.1111/j.1525-1497.2005.0150.x] [Medline: 16050886]

2. Johnson BH, Palmer L, Gatwood J, Lenhart G, Kawai K, Acosta CJ. Annual incidence rates of herpes zoster among an immunocompetent population in the United States. BMC Infect Dis 2015 Nov 06;15:502 [FREE Full text] [doi: 10.1186/s12879-015-1262-8] [Medline: 26546419]

3. Koya KD, Bhatia KR, Hsu JTS, Bhatia AC. YouTube and the expanding role of videos in dermatologic surgery education. Semin Cutan Med Surg 2012 Sep;31(3):163-167 [FREE Full text] [doi: 10.1016/j.sder.2012.06.006] [Medline: 22929353]

4. Lam NHT, Tsiang JT, Woo BKP. Exploring the Role of YouTube in Disseminating Psychoeducation. Acad Psychiatry 2017 Dec;41(6):819-822 [FREE Full text] [doi: 10.1007/s40596-017-0835-9] [Medline: 29022242]

5. Lam NHT, Woo BKP. YouTube as a New Medium for Dementia Education Among Chinese Americans. Community Ment Health J 2020 Apr;56(3):435-439 [FREE Full text] [doi: 10.1007/s10597-019-00493-7] [Medline: 31641910]

6. Guo JZ, Chong KPL, Woo BKP. Utilizing YouTube as platform for psychiatric emergency patient outreach in Chinese Americans. Asian J Psychiatr 2020 Apr;50:101960 [FREE Full text] [doi: 10.1016/j.ajp.2020.101960] [Medline: 32086173]

7. Qi J, Trang T, Doong J, Kang S, Chien AL. Misinformation is prevalent in psoriasis-related YouTube videos. Dermatol Online J 2016 Nov 15;22(11). [Medline: 28329562]

8. Sunderland N, Camm CF, Glover K, Watts A, Warwick G. A quality assessment of respiratory auscultation material on YouTube. Clin Med (Lond) 2014 Aug;14(4):391-395 [FREE Full text] [doi: 10.7861/clinmedicine.14-4-391] [Medline: 25099841]

9. Shires CB, Wilson CD, Sebelik M. Thyroid surgery YouTube videos: estimating quality by surgeon characteristics and view rate. Gland Surg 2019 Jun;8(3):207-211 [FREE Full text] [doi: 10.21037/gs.2018.10.01] [Medline: 31328098]

10. Lenczowski E, Dahiya M. Psoriasis and the Digital Landscape: YouTube as an Information Source for Patients and Medical Professionals. J Clin Aesthet Dermatol 2018 Mar;11(3):36-38 [FREE Full text] [Medline: 29606999]

11. Gray R, Hassanein E, Thompson DR. Journal editors and their h-index. J Adv Nurs 2017 Sep;73(9):2031-2034 [FREE Full text] [doi: 10.1111/jan.13070] [Medline: 27400154]

12. Woo BKP, Chung JOP. Using YouTube analytics to evaluate a Chinese video-based lecture regarding Parkinson's disease. J Clin Neurosci 2018 Apr;50:300 [FREE Full text] [doi: 10.1016/j.jocn.2018.01.001] [Medline: 29361404]

13. Shu S, Woo BKP. The Roles of YouTube and WhatsApp in Dementia Education for the Older Chinese American Population: Longitudinal Analysis. JMIR Aging 2020 Apr 13;3(1):e18179 [FREE Full text] [doi: 10.2196/18179] [Medline: 32281940]

14. Lam NH, Woo BK. Efficacy of Instagram in Promoting Psychoeducation in the Chinese-Speaking Population. Health Equity 2020;4(1):114-116 [FREE Full text] [doi: 10.1089/heq.2019.0078] [Medline: 32258963]

Edited by G Eysenbach; submitted 16.05.20; peer-reviewed by S Shu, J Chung, H Namba, N Lam, E Khoong; comments to author 29.06.20; revised version received 02.07.20; accepted 26.07.20; published 03.09.20

Please cite as:

Dunnsiri T, Kawashita T, Lee SC, Monga AK, Woo BKP

Assessing YouTube as an Educational Tool for Shingles: Cross-Sectional Study

JMIR Dermatol 2020;3(1):e20338

URL: http://derma.jmir.org/2020/1/e20338/

doi: $\underline{10.2196 / 20338}$

PMID:

CTeevit Dunnsiri, Takumi Kawashita, Sharon C Lee, Aaron Kumar Monga, Benjamin K P Woo. Originally published in JMIR Dermatology (http://derma.jmir.org), 03.09.2020. This is an open-access article distributed under the terms of the Creative Commons Attribution License (https://creativecommons.org/licenses/by/4.0/), which permits unrestricted use, distribution, and reproduction in any medium, provided the original work, first published in JMIR Dermatology Research, is properly cited. The complete bibliographic information, a link to the original publication on http://derma.jmir.org, as well as this copyright and license information must be included. 